\title{
The ages of Galactic globular clusters in the context of self-enrichment
}

\author{
T. Decressin ${ }^{1}$, H. Baumgardt ${ }^{1}$, P. Kroupa ${ }^{1}$, G. Meynet ${ }^{2}$ and \\ C. Charbonnel ${ }^{2,3}$ \\ ${ }^{1}$ Argelander Institute for Astronomy (AIfA), Auf dem Hügel 71, D-53121 Bonn, Germany \\ email: decressin@astro.uni-bonn.de, holger@astro.uni-bonn.de, \\ pavel@astro.uni-bonn.de, \\ ${ }^{2}$ Geneva Observatory, University of Geneva, chemin des Maillettes 51, CH-1290 Sauverny, \\ Switzerland \\ email: Georges.Meynet@unige.ch, Corinne.Charbonnel@unige.ch \\ ${ }^{3}$ LATT, CNRS UMR 5572, Université de Toulouse, 14 avenue Edouard Belin, F-31400 \\ Toulouse Cedex 04, France
}

\begin{abstract}
A significant fraction of stars in globular clusters (about 70\%-85\%) exhibit peculiar chemical patterns, with strong abundance variations in light elements along with constant abundances in heavy elements. These abundance anomalies can be created in the H-burning core of a first generation of fast-rotating massive stars, and the corresponding elements are conveyed to the stellar surface thanks to rotational induced mixing. If the rotation of the stars is fast enough, this material is ejected at low velocity through a mechanical wind at the equator. It then pollutes the interstellar medium (ISM) from which a second generation of chemically anomalous stars can be formed. The proportion of anomalous stars to normal stars observed today depends on at least two quantities : (1) the number of polluter stars; (2) the dynamical history of the cluster, which may lose different proportions of first- and second-generation stars during its lifetime. Here we estimate these proportions, based on dynamical models for globular clusters. When internal dynamical evolution and dissolution due to tidal forces are accounted for, starting from an initial fraction of anomalous stars of $10 \%$ produces a present-day fraction of about $25 \%$, still too small with respect to the observed $70-85 \%$. In the case of gas expulsion by supernovae, a much higher fraction is expected to be produced. In this paper we also address the question of the evolution of the second-generation stars that are He-rich, and deduce consequences for the age determination of globular clusters.
\end{abstract}

Keywords. globular clusters: general, stellar dynamics, stellar evolution

\section{Introduction}

It has long been known that globular-cluster stars present some striking anomalies in their content of light elements whereas their heavy elements (i.e., Fe-group, $\alpha$-elements) remain fairly constant from star to star (with the notable exception of $\omega$ Cen). While in all the Galactic globular clusters studied so far one finds "normal" stars with detailed chemical compositions similar to those of field stars of the same metallicity (i.e., same $[\mathrm{Fe} / \mathrm{H}]$ ), one also observes numerous "anomalous" main-sequence and red-giant (RGB) stars that are simultaneously deficient (to various degrees) in $\mathrm{C}, \mathrm{O}$, and $\mathrm{Mg}$, as well as enriched in N, Na, and Al (for reviews see Gratton et al. 2004; Charbonnel 2005).

These abundance variations are expected to result from H-burning nucleosynthesis at high temperatures, around $75 \times 10^{6} \mathrm{~K}$ (Denisenkov \& Denisenkova 1989, 1990; Langer \& Hoffman 1995; Prantzos et al. 2007). Such temperatures are not reached in the lowmass main-sequence and RGB stars that are chemically peculiar, meaning that the stars inherited their abundance anomalies at stellar birth. 


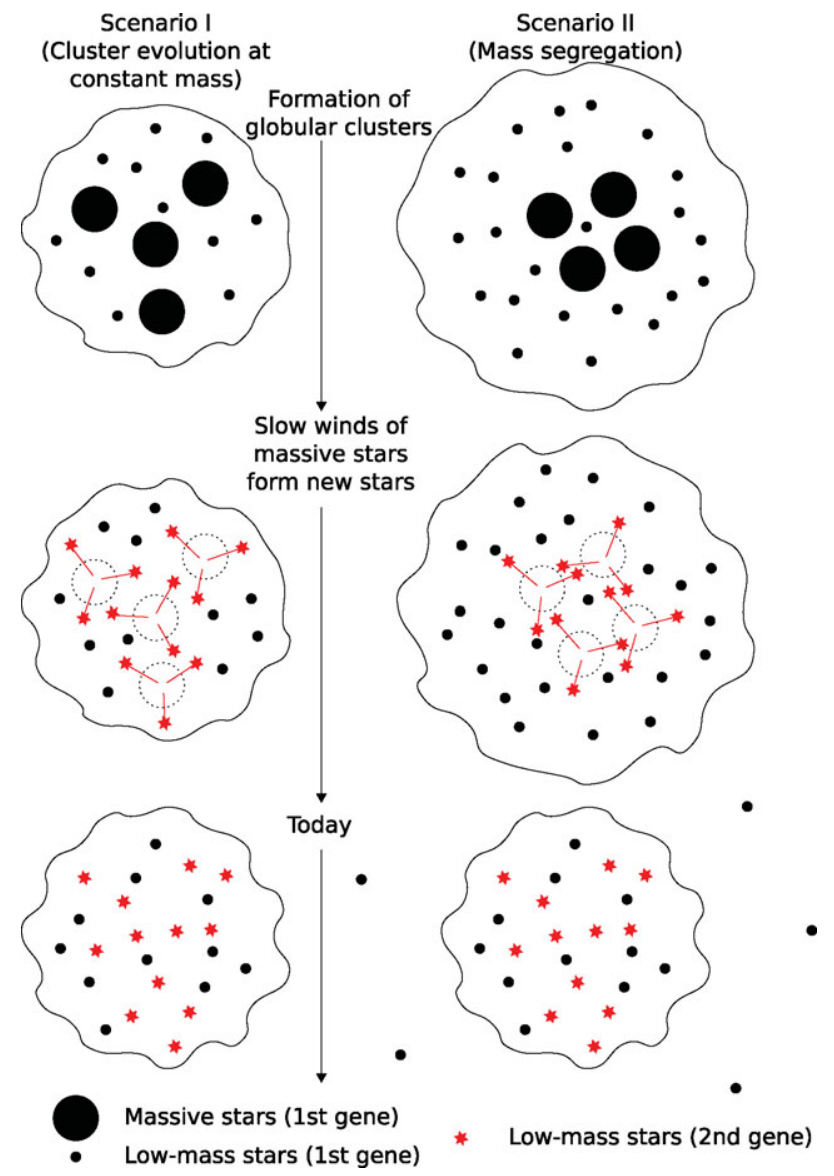

Figure 1. Schematic evolution of a globular cluster: a first generation of stars is born from a giant molecular cloud. Massive stars of the first generation evolve and give birth to a second generation of low-mass stars (dashed symbols in middle panels). Then the cluster evolves and today (lower panels) a mixture of first and second generation low-mass stars is present. In the right panel the cluster is initially mass segregated and massive stars (and hence stars of second generation) are concentrated toward the cluster centre.

Here we follow the work of Prantzos \& Charbonnel (2006) and Decressin et al. (2007b) who propose that abundance anomalies are build up by fast rotating, fast evolving massive stars. During their main sequence evolution, rotationally induced mixing transports elements synthesised in the convective H-burning core to the stellar surface. For stars heavier than $20 \mathrm{M}_{\odot}$, the surface reaches break-up at the equator (i.e., the centrifugal equatorial force balances gravity), provided that their initial rotational velocity is high enough. In such a situation, a slow mechanical wind develops at the equator and forms a disc around the stars, similar to what happens in Be stars (Townsend et al. 2004; Ekström et al. 2008). The material in the discs is strongly enriched in H-burning products and has a slow velocity, allowing it to remain trapped in the potential well of the cluster. On the contrary, matter released later through radiatively driven winds during most of the He-burning phase and then through SN explosions has a very high velocity and is lost from the cluster. Therefore, new stars can form only from the matter available in discs, with the abundance patterns we observe today. Thus globular clusters can contain two populations of low-mass stars: a first generation which has the chemical composition 
of the material out of which the cluster formed (similar to field stars with similar metallicity); and a second generation of stars harbouring the abundance anomalies born from the ejecta of fast-rotating massive stars. This scenario is sketched in Fig. 1.

\section{Dynamical issues}

\subsection{The number ratio between the two populations in globular clusters}

Based on the determination of the compositions of giant stars in NGC 2808 by Carretta et al. (2006), Prantzos \& Charbonnel (2006) determined that around $70 \%$ of stars show abundance anomalies in this specific cluster today. Decressin et al. (2007a) find similar results for NGC 6752 with their analysis of the data of Carretta et al. (2007): around 85\% of the cluster stars (of the sample of 120 stars) present abundance anomalies. Therefore most stars still evolving in globular clusters seem to be second-generation stars.

How can one produce such a high fraction of chemically peculiar stars? The main problem is that, assuming a Salpeter (1955) IMF for the polluters, the accumulated mass of the slow winds ejected by the fast-rotating massive stars would only provide $10 \%$ of the total number of low-mass stars. To match the observations thus requires either (a) a flat IMF with a slope of 0.55 instead of 1.35 (Salpeter's value), or (b) that $95 \%$ of the first generation stars have escaped the cluster (Decressin et al. 2007a). Here we first investigate whether such a high loss of stars is possible, and what are the main processes that could drive it.
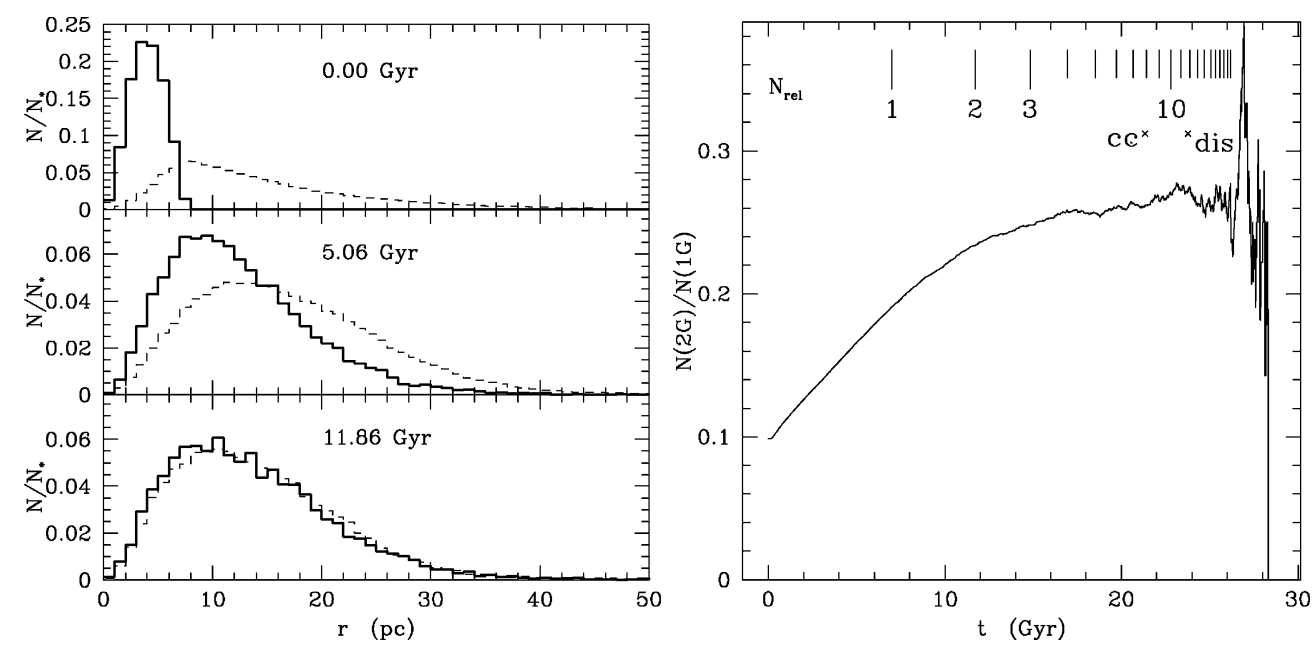

Figure 2. Left: radial distribution of the first (dashed lines) and second (full lines) generation of low-mass stars at three different times. Each histogram is normalised to the total number of stars in each population. Right: Number ratio between the second (with low initial specific energy) and first (with high initial specific energy) population of low-mass stars in a cluster with initially $128 \mathrm{~K}$ stars as a function of time. At the top of each panel the number of elapsed relaxation times, with crosses indicating the times of core collapse and of cluster dissolution.

\subsection{Dynamical evolution of globular clusters}

First we assume that the globular clusters display primordial mass segregation so that the massive stars are located at their center. Since we expect that the formation of the second generation of low-mass stars happens locally around individual massive stars (see Decressin et al. 2007a for more details), the second generation of stars will also be 
initially more centrally concentrated than the first generation. In such a situation, two competitive processes act in the clusters: the loss of stars in the outer parts of the cluster will first reduce the number of bound first-generation stars; and the dynamical spread of the initially more concentrated second-generation stars will stop this differential loss when the two populations are dynamically mixed.

Our analysis, based on the N-body models computed by Baumgardt \& Makino (2003) with the collisional Aarseth N-body code NBODY4 (Aarseth 1999), is presented in detail in Decressin et al. (2008).

As these models have been computed for a single stellar population, we apply the following process to mimic the formation of a cluster with two dynamically distinct populations: we sort all the low-mass stars $\left(M \leqslant 0.9 \mathrm{M}_{\odot}\right)$ according to their specific energy (i.e., their energy per unit mass). We define the second stellar generation as the stars with lowest specific energies, (i.e., those which are most tightly bound to the cluster due to their small central distance and low velocity). The number of second-generation stars is given by having their total number representing $10 \%$ of the total number of low-mass stars.

In Fig. 2 one can see the radial distribution of the two populations at various epochs. Initially, first-generation stars show an extended distribution up to $40 \mathrm{pc}$ whereas the second-generation stars (with low specific energy) are concentrated within 6 pc around the centre.

Progressively the second-generation stars spread out due to dynamical encounters so their radial distribution extends. However this process operates on a long timescale: even after 5 Gyr of evolution the two populations still have different distributions. The bottom panel of Fig. 2 shows that after nearly 12 Gyr of evolution (slightly less than 3 elapsed relaxation times) the two populations have similar radial distributions and can no longer be distinguished owing to their dynamical properties.

As previously seen, the effect of the external potential of the Galaxy on the cluster is to strip away stars lying in the outer part of the cluster. Initially, as only stars of the first generation populate the outer part of the cluster owing to their high specific energy, only these first-generation stars are lost in the early times. This lasts until the second-generation stars migrate towards the outer part of the cluster. Depending on the cluster mass, it takes between 1 to 4 Gyr to start losing second-generation stars. Due to the time-delay to lose second-generation stars, their remaining fraction in the cluster is always higher than that of the first-generation stars, except during the final stage of cluster dissolution. Fig. 2 (right panel) quantifies this point by showing the time evolution of the number ratio of second to first-generation stars. As a direct consequence of our selection procedure, the initial ratio is 0.1 ; it then increases gradually with time and it tends to stay nearly constant as soon as the two distributions are similar. Finally, at the time of cluster dissolution (i.e., when the cluster has lost $95 \%$ of its initial mass, indicated by the label "dis" in Fig. 2), large variations occur due to the low number of low-mass stars present in the cluster. In Fig. 2 (right panel) we have also indicated the number of elapsed relaxation times, showing that the increase of the number ratio lasts only 3 relaxation times.

The fraction of second-generation stars relative to first-generation ones increases by a factor of 2.5 over the cluster history. Therefore, these second-generations stars can account for $25 \%$ of the low-mass stars present in the clusters. Compared to the observed ratios (70\% and $85 \%$ in NGC 2808 and NGC 6752 respectively) the internal dynamical evolution and the dissolution due to the tidal forces of the host Galaxy are not efficient enough. An additional mechanism is thus needed to expel the first-generation stars more effectively. 


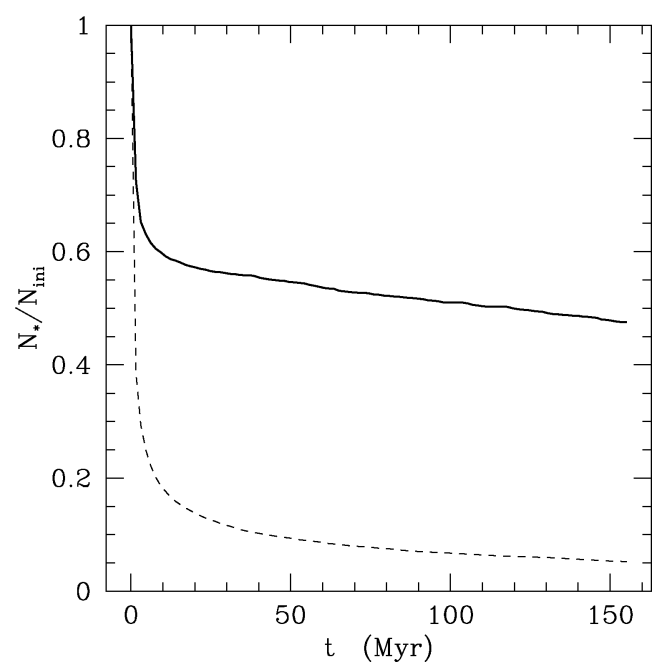

Figure 3. Evolution of the fraction of first- (dashed line) and second-generation (full line) stars still bound to the cluster with initial parameters: SFE of $0.33, r_{h} / r_{t}=0.06$ and $\tau_{M} / t_{\text {Cross }}=0.33$.

\subsection{Gas expulsion}

As it operates early in the cluster history (a few million years after cluster formation at the latest), initial gas expulsion by supernovae is an ideal candidate for such a process. As the gas still present after the star formation is quickly removed, it ensues a strong lowering of the potential well of the cluster so that the outer parts of the cluster can become unbound.

Baumgardt \& Kroupa (2007) computed a grid of N-body models to study this process and its influence on cluster evolution by varying the free parameters: star formation efficiency, SFE, ratio between the half-mass and tidal radius, $r_{h} / r_{t}$, and the ratio between the timescale for gas expulsion to the crossing time, $\tau_{M} / t_{\text {Cross }}$. They show in particular that, in some extreme cases, the complete disruption of the cluster can be induced by gas expulsion. This process has also been used successfully by Marks et al. (2008) to explain the challenging correlation between the central concentration and the mass function of globular clusters as found by De Marchi et al. (2007).

We have applied the same method as the one we used in $\S 2.2$ to the models of Baumgardt \& Kroupa (2007). Fig. 3 shows that in the case of a cluster which loses around $90 \%$ of its stars, the ejection of stars from the cluster mostly concerns firstgeneration ones. At the end of the computation only $5 \%$ of the first-generation stars remain bound to the cluster, along with around half of the second-generation stars. Therefore the number ratio of second to first-generation stars increases by a factor of 10 : half of the population of low-mass stars still populating the cluster are second-generation stars. Further, the initial radial distribution is not totally erased by this mechanism, as the second-generation stars are still more centrally distributed. We can expect that this ratio will continue to increase during the long-term evolution of the cluster (see Decressin et al., in preparation).

Thus if globular clusters are born mass segregated, dynamical processes (gas expulsion, tidal stripping and two-body relaxation) can explain the number fraction of secondgeneration stars with abundance anomalies. Similar conclusions have been reached by D'Ercole et al. (2008). 

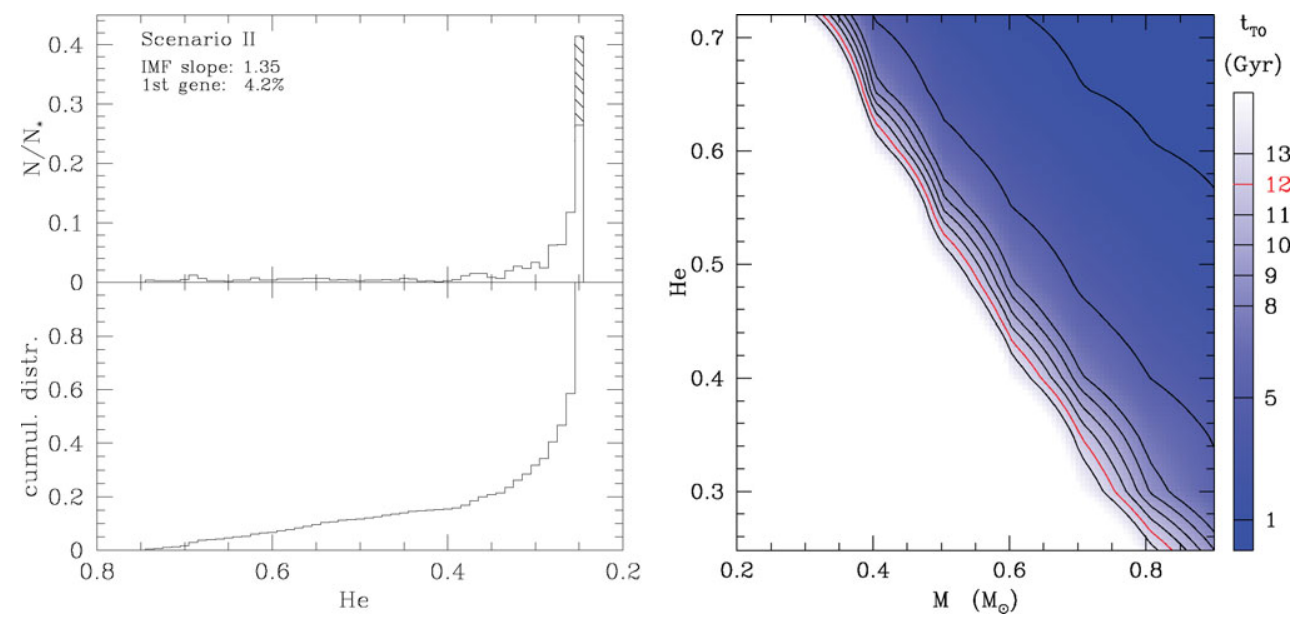

Figure 4. Left: distribution function of He for low-mass stars of second (white area) and first (hatched area) generation (top panel) and the cumulative distribution function of He in low mass-stars (bottom panel). Right: Age at the turn-off for low-mass stars as a function of their mass $\left(0.2-0.9 \mathrm{M}_{\odot}\right)$ and initial He value $(0.245-0.72$, mass fraction). White area indicates stars still on the main sequence after 15 Gyr of evolution.

\section{He-rich stars}

Since the abundance variations in light elements are expected to be due to H-burning whose direct product is Helium, we expect that second-generation stars are also enriched in $\mathrm{He}$, to some degree. Unfortunately the He abundance cannot be directly measured in globular-cluster stars and we have only indirect evidence for an overabundance of He. The globular clusters $\omega$ Cen and NGC 2808 display multiple main sequences (Piotto et al. 2005, 2007); a double sub-giant branch is also found in NGC 1851 (Milone et al. 2008). Such features can be understood if the stars have a range of He contents. He enrichment is also a possible explanation for the appearance of extreme horizontal branches, as seen in several globular clusters (Caloi \& D'Antona 2005, 2007).

\subsection{Evolution of He-rich stars}

As explained in Decressin et al. (2007a), material stored in the discs around massive stars is heavily enriched with He. Fig. 4 (left panel) gives our expected theoretical distribution function of the He-value in low-mass stars in NGC 6752. A main peak is present at $Y=0.245$ and it extends up to 0.4 . However a long tail toward higher Y-values is also present with around $12 \%$ of the stars with initial He value between 0.4 and 0.72 .

To assess the implications for globular clusters induced by this population of Herich stars we have computed a grid of low-mass stellar models from 0.2 to $0.9 \mathrm{M}_{\odot}$ at a metallicity of $Z=0.0005$ (similar to the metal-poor globular cluster NGC 6752) for initial He mass fraction between 0.245 and 0.72 with the stellar evolution code STAREVOL V2.92 (see Siess et al. 2000; Siess 2006, for more details). These models have been computed without any kind of mixing except for an instantaneous mixing in convection zones. The adopted mass-loss rate follows the Reimers (1975) prescription (with the parameter value $\left.\eta_{\mathrm{R}}=0.5\right)$ with a $\sqrt{Z / Z_{\odot}}$ dependence. All models have been computed from the pre-main sequence phase to the end of the central He-burning phase.

For a given stellar mass, He-rich stars evolve faster on the main-sequence due to their lower initial H-content and to their higher luminosity. Figure 4 illustrates this point showing the turn-off age as a function of the initial mass and He mass fraction of stars. 

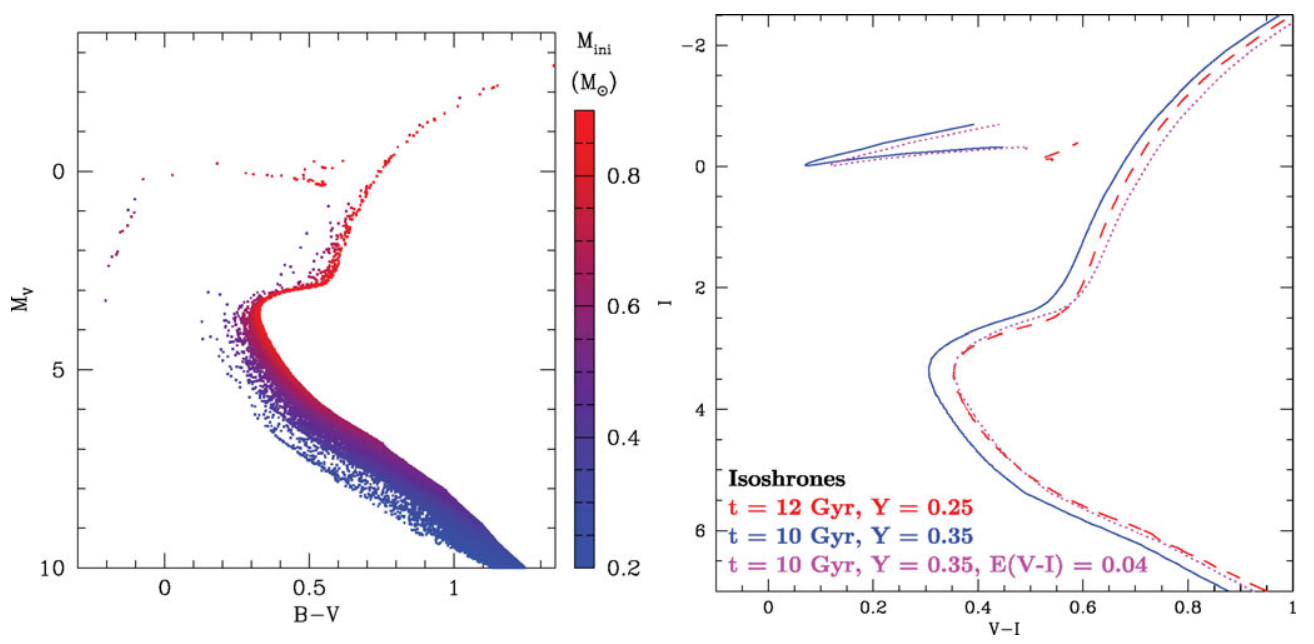

Figure 5. Left: Colour-magnitude diagram of a 12 Gyr old globular clusters with initial spread in He in stars similar to Fig. 4 (left panel). Right: isochrone of 12 Gyr and initial He of 0.25 (dashed lines) and isochrones of $10 \mathrm{Gyr}$ and initial He of 0.35 without (full lines) and with (dotted lines) additional reddening.

After 12 Gyr, stars of $0.85 \mathrm{M}_{\odot}$ with standard helium $(Y=0.245)$, as well as He-rich stars of $0.4 \mathrm{M}_{\odot}(Y=0.6)$, are leaving the main sequence.

\subsection{Effects on globular clusters}

Figure 5 (left panel) shows a synthetic colour-magnitude diagram (CMD) of a 12 Gyr old globular cluster with an initial spread in He as given by Fig. 4 (left panel). This CMD has been computed with a modified version of the program used by Meynet (1993) to investigate supergiant populations. The spread in He converts into a spread in mass at the turn-off. The luminosity increase of He-rich stars is mainly compensated by their shorter lifetime so that the turn-off luminosity is almost constant. In addition, due to their differences in opacity and to their compactness, they are also hotter. Thus the Herich main-sequence and RGB stars are shifted to the left side of the CMD. They also occupy the blue part of the HB down to the extreme-HB location.

If we compare our theoretical CMD with the one observed by Brown et al. (2005) for NGC 6752 we note some discrepancies. First the theoretical width of the main sequence at the turn-off is too large for this cluster. Additionally NGC 6752 shows an extended blue HB, with no stars in the red part. This last discrepancy could be attributed to the low mass-loss rate used in the stellar models which do not remove enough mass along the RGB and hence produce stars on the HB that are too cool. As the theoretical spread of the initial He is strongly affected by the dilution of the disc ejected by fast rotating massive stars and the ISM, we plan to constrain this dilution with the observed sequences to check whether we are able to consistently reproduce both the abundance anomalies and the He-value inferred in globular clusters (see Decressin et al., in preparation).

The uncertainties pertaining the ages of globular clusters are manifold. Among them, photometric uncertainties widen both sides of the main-sequence, unresolved binaries broaden the main-sequence towards cooler effective temperature, an increase of metallicity (as seen in $\omega$ Cen) induces redder sequences. The presence of He-rich stars can induce some additional uncertainties. Let us note that the He content is the only physical parameter which broadens the main-sequence only to its left (i.e., blue) side. In Fig. 5 (right panel) we evaluate uncertainties related to He-rich stars: we try to reproduce a 
He-normal isochrone $(Y=0.25)$ with a He-rich $(Y=0.35)$ one. This could be done with an isochrone 2 Gyr younger along with an increase of the reddening of 0.04 magnitudes. The differences between the He-rich with reddening and He-normal isochrones are small around the TO, the main-sequence and the subgiant-branch. Discrepancies appear along the RGB and at the level of the HB, where the normal He-rich isochrone is much less extended toward the blue. Thus the age uncertainties due to a population of He-rich stars can be of the order of 1-2 Gyr.

\section{Acknowledgements}

T. D. and C. C. acknowledges financial support from the Swiss FNS.

\section{References}

Aarseth, S. J. 1999, PASP, 111, 1333

Baumgardt, H. \& Kroupa, P. 2007, MNRAS, 380, 1589

Baumgardt, H. \& Makino, J. 2003, MNRAS, 340, 227

Brown, T. M., Ferguson, H. C., Smith, E., et al. 2005, AJ, 130, 1693

Caloi, V. \& D'Antona, F. 2005, A\&A, 435, 987

Caloi, V. \& D'Antona, F. 2007, A\&3A, 463, 949

Carretta, E., Bragaglia, A., Gratton, R. G., et al. 2006, A\&SA, 450, 523

Carretta, E., Bragaglia, A., Gratton, R. G., Lucatello, S., \& Momany, Y. 2007, A $\varangle A$, 464, 927

Charbonnel, C. 2005, in IAU Symposium 228, ed. V. Hill, P. François, \& F. Primas, 347

De Marchi, G., Paresce, F., \& Pulone, L. 2007, ApJl, 656, L65

Decressin, T., Baumgardt, H., \& Kroupa, P. 2008, A\& A, in press

Decressin, T., Charbonnel, C., \& Meynet, G. 2007a, A\&A, 475, 859

Decressin, T., Meynet, G., Charbonnel, C., Prantzos, N., \& Ekström, S. 2007b, A\& A, 464, 1029

Denisenkov, P. A. \& Denisenkova, S. N. 1989, Astronomicheskij Tsirkulyar, 1538, 11

Denisenkov, P. A. \& Denisenkova, S. N. 1990, Soviet Astronomy Letters, 16, 275

D'Ercole, A., Vesperini, E., D'Antona, F., McMillan, S. L. W., \& Recchi, S. 2008, MNRAS, 1228

Ekström, S., Meynet, G., Maeder, A., \& Barblan, F. 2008, A\&A, 478, 467

Gratton, R., Sneden, C., \& Carretta, E. 2004, ARA\&A, 42, 385

Langer, G. E. \& Hoffman, R. D. 1995, PASP, 107, 1177

Marks, M., Kroupa, P., \& Baumgardt, H. 2008, MNRAS, 386, 2047

Meynet, G. 1993, in The Feedback of Chemical Evolution on the Stellar Content of Galaxies, ed. D. Alloin \& G. Stasińska, 40

Milone, A. P., Bedin, L. R., Piotto, G., et al. 2008, ApJ, 673, 241

Piotto, G., Bedin, L. R., Anderson, J., et al. 2007, ApJl, 661, L53

Piotto, G., Villanova, S., Bedin, L. R., et al. 2005, ApJ, 621, 777

Prantzos, N. \& Charbonnel, C. 2006, A\&SA, 458, 135

Prantzos, N., Charbonnel, C., \& Iliadis, C. 2007, A\&A A, 470, 179

Reimers, D. 1975, Circumstellar envelopes and mass loss of red giant stars (Problems in stellar atmospheres and envelopes.), 229

Salpeter, E. E. 1955, ApJ, 121, 161

Siess, L. 2006, $A \mathscr{E} A$, 448, 717

Siess, L., Dufour, E., \& Forestini, M. 2000, A\&A, 358, 593

Townsend, R. H. D., Owocki, S. P., \& Howarth, I. D. 2004, MNRAS, 350, 189 


\section{Discussion}

A. Bragaglia: Have you also tried to reproduce NGC 2808 with your models? You predict a continuous He enhancement, while from the three sequences we require discrete values.

T. Decressin: Actually I have used a local dilution of the slow winds produced by fast-rotating massive stars with the intra-cluster medium to be able to reproduce the continuous distribution of $[\mathrm{O} / \mathrm{Na}]$ in NGC 6752 . This scheme obviously leads to a continuous He-enrichment, contrary to what is observed in NGC 2808. In the future I will try to use this discrete He value to constrain the dilution process, which is presently poorly understood. 


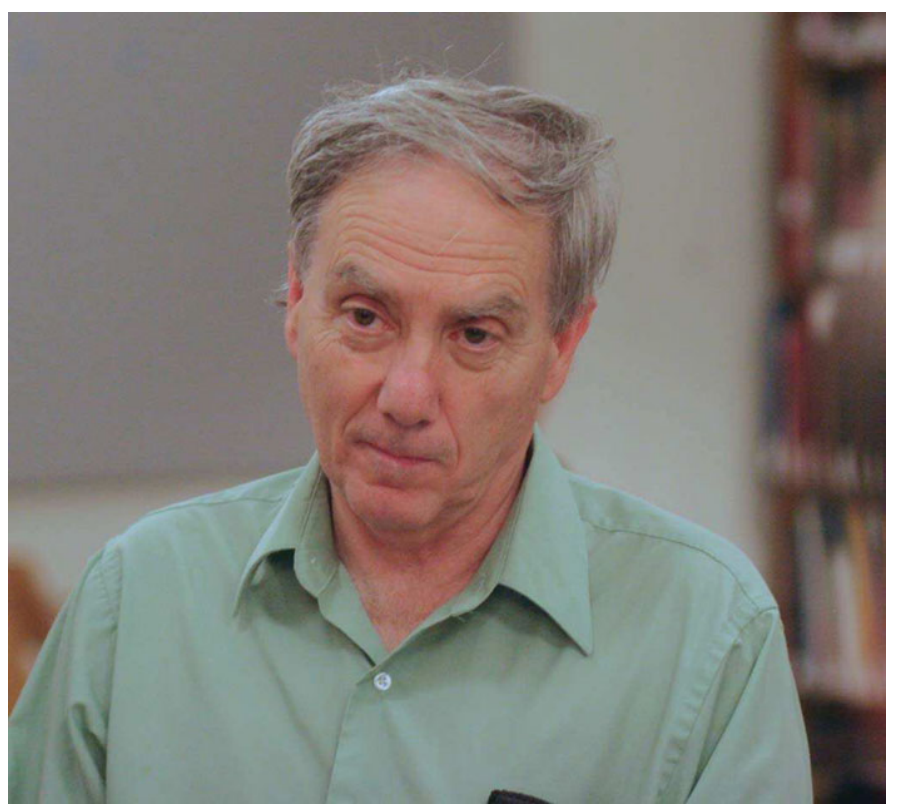

Mario Livio

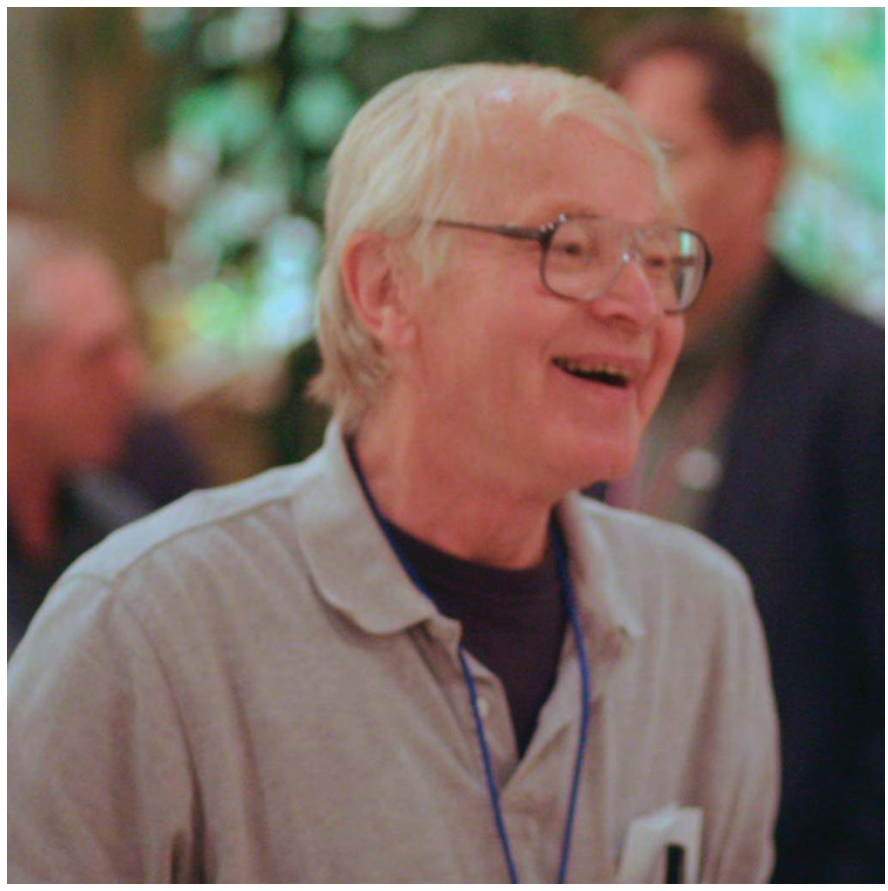

Michal Simon 